\title{
Preface SSW 2011
}

\author{
Paolo Cappellari ${ }^{1}$, Roberto De Virgilio ${ }^{2}$, and Mark Roantree ${ }^{1}$ \\ 1 Dublin City University, Dublin, Ireland \\ \{pcappellari,mark\}@computing.dcu.ie \\ 2 Universitá Roma Tre, Rome, Italy \\ dvr@dia.uniroma3.it
}

We are witnessing a smooth evolution of the Web from a worldwide information space of linked documents to a global knowledge base, composed of semantically interconnected resources. To date, the correlated and semantically annotated data available on the web amounts to 25 billion RDF triples, interlinked by roughly 395 million RDF links. The continuous publishing and the integration of semantic datasets from companies, government and public sector projects is leading to the creation of the so-called Web of Knowledge. Each semantic dataset contributes to extend the global knowledge and increases its reasoning capabilities. Researchers are now looking with growing interest to semantic issues in this huge amount of correlated data available on the Web. Many progresses have been made in the field of semantic technologies, from formal models to repositories and reasoning engines. While many practitioners focus on exploiting semantic information to contribute to IR problems from a document centric point of view, we believe that such a vast, and constantly growing, amount of semantic data raises data management issues that must be faced in a dynamic, highly distributed and heterogeneous environment such as the Web.

The SSW workshop was organized by researchers at Universitá Roma Tre and Dublin City University to provide a forum for discussion and advancement of data management in searching the web, and in relationships with semantic web technologies that propose new models, languages and applications. The workshop has four full research papers and one poster paper. Ferre et al. introduce semantic faceted search, a combination of an expressive query language and faceted search to improve the query process for end users. Schuller and Weinzierl offer semantic reasoning in the area of multi-context systems with a top-down approach with no imposition on the context of either logic or syntax. A collaborative framework for annotating images in semantic search is presented in the paper by Hong and Reiff-Marganiec. Tian et al. address the issue of homonym search, developing a new interface for an ontology-supported web search. In the short poster paper, McGinnes presents a semantic strategy to exploit ontologies for image retrieval in conceptual modeling.

We wish to express special thanks to the Program Committee members for helping us prepare an interesting program and express our appreciation to the authors of the papers for sharing their work with us. We thank the CAiSE 2011 
organizers for their help and organizational support. Finally, we would like to extend many thanks to Universitá Roma Tre and to Dublin City University for their support in organizing the workshop.

April 2011

Paolo Cappellari Roberto De Virgilio Mark Roantree 


\section{Organization}

\section{Program Committee Chairs}

Paolo Cappellari

Mark Roantree

Roberto De Virgilio

\section{Program Committee}

François Bry

Paolo Cappellari

Vassilis Christophides

Simona Colucci

Roberto De Virgilio

Beniamino Di Martino

Tommaso Di Noia

Bettina Fazzinga

Flavius Frasincar

Tim Furche

James Geller

Laura Hollink

Clemens Ley

Dimitris Plexousakis

Mark Roantree

Michele Ruta

Michael Schmidt

Luciano Serafini

Eufemia Tinelli

Kees Van Der Sluijs

Antonius Weinzierl
Dublin City University, Ireland Dublin City University, Ireland Universitá Roma Tre, Italy

University of Munich, Germany

Dublin City University, Ireland

University of Crete, Greece

Technical University of Bari, Italy

Universitá Roma Tre, Rome, Italy

Second University of Naples, Italy

Technical University of Bari, Italy

University of Calabria, Italy

Erasmus University Rotterdam, Netherlands

University of Munich, Munich, Germany

New Jersey Institute of Technology, U.S.A.

Delft University of Technology, Netherlands

Oxford University, U.K.

University of Crete, Greece

Dublin City University, Ireland

Technical University of Bari, Italy

University of Freiburg, Germany

FBK-IRST, Italy

Technical University of Bari, Italy

Technische Universiteit Eindhoven, Netherlands

Vienna University of Technology, Austria 